\title{
MAXIMUM TORQUE AND MOMENTUM ENVELOPES FOR REACTION WHEEL ARRAYS
}

\author{
R. G. Reynolds \\ SpectrumAstro, Inc., Herndon, VA \\ F. Landis Markley \\ NASA's Goddard Space Flight Center, Greenbelt, MD
}

\begin{abstract}
Spacecraft reaction wheel maneuvers are limited by the maximum torque and/or angular momentum which the wheels can provide. For an $n$-wheel configuration, the torque or momentum envelope can be obtained by projecting the $n$ dimensional hypercube, representing the domain boundary of individual wheel torques or momenta, into three dimensional space via the $3 \times n$ matrix of wheel axes. In this paper, the properties of the projected hypercube are discussed, and algorithms are proposed for determining this maximal torque or momentum envelope for general wheel configurations. Practical implementation strategies for specific wheel configurations are also considered.
\end{abstract}

\section{INTRODUCTION}

Many spacecraft employ more than three wheels for attitude control, both for redundancy and for the additional maneuvering capability of the extra wheels. The Swift Gamma Ray Burst Explorer, for example, will employ six reaction wheels for rapid slewing to enable observation of the initial stages of ephemeral gamma ray burst events ${ }^{1}$. In order to employ these wheels effectively, it is necessary to characterize the maximum torque available in any direction from the reaction wheels. Since long slews may be momentum-limited rather than torque-limited, it is equally necessary to characterize the maximum angular momentum storage capability in any direction. This knowledge is also important in designing momentum unloading strategies. Fortunately, the mathematical analyses required to identify the maximum torque and maximum angular momentum storage are identical. This analysis is the subject of this paper. Previous work on the optimal use of more than three reaction wheels has largely been limited to the four-wheel case $\mathrm{e}^{2-4}$.

In the case of equal wheel capabilities, the available torques fill the interior of an $n$-dimensional hypercube centered on the origin in reaction wheel torque space. If the torque capabilities vary from wheel to wheel, the torques fill the interior of an $n$-dimensional rectangular parallelopiped in this space, where each side has length equal to twice the magnitude of the maximum torque that a given wheel can provide. We will refer to this figure as a hypercube for brevity, at the expense of precision, since the case of equal wheel capabilities is the most common in practice.

The available torques in three dimensional space fill a polyhedron given by the mapping of this hypercube by the $3 \times n$ reaction wheel torque distribution matrix, whose columns are the unit vectors parallel to the reaction wheel axes in three dimensional space. We will only consider "non-defective" reaction wheel configurations, by which we mean that no three of the reaction wheel spin axis directions are coplanar. As shown below, this polyhedron is the convex hull of the mapping of the vertices of the $n$-dimensional hypercube, which are the points at which all $n$ wheels are saturated in the positive or negative direction. The exterior vertices of the three-dimensional envelope are connected by edges that are the boundaries of the two dimensional facets that form the envelope.

We have developed algorithms for defining the envelope and for finding the maximum torque in a given direction. The latter algorithm examines all the facets of the envelope to determine which facet limits the maximum torque, and hence which $n-2$ wheels have saturated torque commands. It is then straightforward to find the remaining two wheel torque commands necessary to produce the desired net torque. While the exact solution algorithm is not very efficient for onboard processing, it works for arbitrary wheel configurations. When the wheel configuration is known, it is possible to develop much more efficient algorithms. 


\section{GEOMETRY OF THE ENVELOPE}

\section{General Analysis}

For brevity of exposition, we will only refer to torques in the following; the analysis for angular momentum is identical. The torques of an $n$-wheel system can be represented by an $n$-dimensional vector

$$
\mathbf{T}_{\text {wheels }}=\left[T_{1} T_{2} \cdots T_{n}\right]
$$

These torques fill the interior of an $n$-dimensional hypercube, where the length of the side along the $i$ th axis is two times the maximum torque magnitude $T_{\max i}$ of the wheel along that axis. This can be the absolute maximum that the wheel can provide, or it can be some desired maximum for a given attitude maneuver. The vertices of the hypercube are points where all wheels are saturated, i.e., they supply either their positive or negative maximum torques. Each edge has all but one wheel saturated and is parallel to the axis of the unsaturated wheel. The facets of the hypercube have all but two wheels saturated.

The torque in three dimensional space is

$$
\mathbf{T}=W \mathbf{T}_{\text {wheels }}
$$

where

$$
W \equiv\left[\hat{\mathbf{w}}_{1} \hat{\mathbf{w}}_{2} \cdots \hat{\mathbf{w}}_{n}\right]
$$

is the torque distribution matrix. The available torques in three dimensional space fill a polyhedron specified by the mapping of the $n$-dimensional hypercube by Eq. (2). The vertices, edges, and facets of the polyhedron in three dimensional space are mappings of the vertices, edges, and facets of the hypercube. However, this mapping is not 1:1, since some of the vertices, edges, and facets of the hypercube will map to the interior of the polyhedron in three-dimensional space. Thus the vertices of the available polyhedron in three dimensional space are points where all wheels are saturated. The edges of the polyhedron have all but one wheel saturated, and an edge with wheel $i$ unsaturated is parallel to the wheel's spin axis $\hat{\mathbf{w}}_{i}$. The facets have all but two wheels saturated, and the facet with wheels $i$ and $j$ unsaturated is a parallelogram with sides parallel to $\hat{\mathbf{w}}_{i}$ and $\hat{\mathbf{w}}_{j}$ and with normal vector in the direction of the cross product $\hat{\mathbf{w}}_{i} \times \hat{\mathbf{w}}_{j}$.

We can identify the bounding planes of the polyhedron, and thus determine the envelope, by considering all pairs $i, j$ of wheels in turn. There are $n(n-1) / 2$ such pairs for an $n$-wheel configuration. The $i, j$ pair defines a set of $2^{n-2}$ facets of the hypercube in the $n$ dimensional reaction wheel command space. Each facet corresponds to a saturated command for the $n-2$ wheels other than wheels $i$ and $j$, and with the torque commands for wheels $i$ and $j$ varying over their full range over the facet. Under the transformation into three dimensional space specified by Eq. (2), these facets map onto $2^{n-2}$ parallel planes with normal vector

$$
\hat{\mathbf{n}}_{i j} \equiv\left(\hat{\mathbf{w}}_{i} \times \hat{\mathbf{w}}_{j}\right) /\left|\hat{\mathbf{w}}_{i} \times \hat{\mathbf{w}}_{j}\right| .
$$

On each of these planes, the wheel torque in the direction of the normal to the plane is

$$
\mathbf{T} \cdot \hat{\mathbf{n}}_{i j}=\sum_{k=1}^{n} T_{k}\left[\hat{\mathbf{w}}_{k} \cdot\left(\hat{\mathbf{w}}_{i} \times \hat{\mathbf{w}}_{j}\right)\right] /\left|\hat{\mathbf{w}}_{i} \times \hat{\mathbf{w}}_{j}\right|=\sum_{k \neq i, j} \sigma_{k} T_{\max k}\left[\hat{\mathbf{w}}_{k} \cdot\left(\hat{\mathbf{w}}_{i} \times \hat{\mathbf{w}}_{j}\right)\right] /\left|\hat{\mathbf{w}}_{i} \times \hat{\mathbf{w}}_{j}\right|,
$$

where $\sigma_{k}= \pm 1$ specifies the direction of saturation of the torque on wheel $k$. Wheels $i$ and $j$ can clearly be omitted from the sum, since they don't contribute to the torque perpendicular to the plane. This is indicated algebraically by the vanishing of the triple product $\hat{\mathbf{w}}_{k} \cdot\left(\hat{\mathbf{w}}_{i} \times \hat{\mathbf{w}}_{j}\right)$ for $k=i$ or $j$. This triple product does not vanish for any other $k$, since we have assumed a "non-defective" reaction wheel configuration, for which no three of the reaction wheel spin axis directions are coplanar.

The planes with the maximum and minimum torque in the direction of the normal are the planes for which

$$
\sigma_{k}=\operatorname{sign}\left[\hat{\mathbf{w}}_{k} \cdot\left(\hat{\mathbf{w}}_{i} \times \hat{\mathbf{w}}_{j}\right)\right]
$$

or

$$
\sigma_{k}=-\operatorname{sign}\left[\hat{\mathbf{w}}_{k} \cdot\left(\hat{\mathbf{w}}_{i} \times \hat{\mathbf{w}}_{j}\right)\right]
$$


for all $k$ other than $i$ and $j$. It is clear that these two planes and only these of the $2^{n-2}$ parallel planes for the $i, j$ pair form part of the torque envelope. On each of these bounding facets $T_{i}$ and $T_{j}$ vary over the range between $\pm T_{\max i}$ and $\pm T_{\max ;}$, respectively. Saturation of $T_{i}$ and $T_{j}$ give the four corners of a parallelogram, so we can identify eight of the vertices of the envelope for each $i, j$ pair, corresponding to the two signs each for saturated $T_{i}$ and $T_{j}$, and the overall sign of the other saturated wheel torques given by Eq. (6a) or (6b).

Carrying out the above procedure over all pairs $i, j$ will produce a total of $4 n(n-1)$ vertices. These aren't all distinct, however, for each vertex will be identified once for each bounding plane of the envelope that intersects there. This is useful information about the "structure" of the envelope, where "structure" denotes the number of vertices of the envelope, and the number of planes and edges that intersect at each vertex, as well as the specific information about which wheels are saturated in which direction at each vertex. It is interesting that this structure depends only on the unit vectors through their triple products $\hat{\mathbf{w}}_{k} \cdot\left(\hat{\mathbf{w}}_{i} \times \hat{\mathbf{w}}_{j}\right)$, and not on the maximum torque capabilities of the wheels. If this torque capability is changed, the shape of the envelope will change, but not the structure as defined here. It is obvious that if the envelope has an inversion symmetry; for each vertex of torque $\mathbf{T}$ there is a corresponding vertex of torque $-\mathbf{T}$ obtained by reversing all the signs $\sigma_{k}$.

\section{Specific Cases}

First consider the easy case of three wheels. The envelope has the structure of a cube, with three planes meeting at each vertex. Thus each vertex will be identified three times by the procedure described above, and the number of distinct vertices is $4 \cdot 3 \cdot(3-1) / 3=8$, the correct number of vertices of a cube. Figure 1 shows the torque envelope for three orthogonal wheels with equal torque capabilities. For this case, the wheel torque distribution matrix $W$ is the $3 \times 3$ identity matrix and the envelope is actually a cube. The unit of length in this and subsequent figures is the maximum torque capability of a single wheel. The momentum envelope, scaled to the maximum momentum storage capability of a single wheel, is identical. Since the figures represent both envelopes, they are labeled Envelope, rather than Torque Envelope or Momentum Envelope.

We next consider the often-employed case of four reaction wheels are oriented in a tetrahedral configuration around the $x$ axis. The torque distribution matrix is

$$
W=\left[\begin{array}{cccc}
s & s & s & s \\
0 & c & 0 & -c \\
c & 0 & -c & 0
\end{array}\right]
$$

where $s \equiv \sin \eta$ and $c \equiv \cos \eta$, with $\eta$ being an angle chosen based on convenience of fabrication or on spacecraft inertia properties. The torque or momentum envelope for this configuration, again assuming identical wheels, is shown in Figure 2.

The next case we consider has the wheels oriented with hexagonal symmetry around the $x$ axis. This is a good approximation to the reaction wheel configuration on the Swift spacecraft, whose moment of inertia is approximately axially symmetric about the $x$ axis. The torque distribution matrix is

$$
W=\left[\begin{array}{cccccc}
s & s & s & s & s & s \\
0 & \frac{\sqrt{3}}{2} c & \frac{\sqrt{3}}{2} c & 0 & -\frac{\sqrt{3}}{2} c & -\frac{\sqrt{3}}{2} c \\
c & \frac{1}{2} c & -\frac{1}{2} c & -c & -\frac{1}{2} c & \frac{1}{2} c
\end{array}\right] .
$$

This six-wheel configuration has an envelope with 32 vertices as shown in Table 1 . Two of the vertices are defined by the intersection of six facets each, 18 by four facets each, and 12 by three facets each. The index pairs labeling the facets are the indices of the pair of wheels that are not saturated on the facet. Figure 3 illustrates the torque or momentum envelope for six wheels with identical capabilities in this configuration. This envelope has a sixfold rotational symmetry about the $\mathrm{z}$ axis.

Finally, Figure 4 shows the torque or momentum envelope for the case where the torque distribution matrix is obtained by deleting one column from the matrix of Eq. (8). This illustrates the case of a six-wheel configuration with one failed wheel. This case will be discussed further below. 
Table 1. Vertices of envelope for the six-wheel configuration

\begin{tabular}{|c|c|c|c|c|c|c|}
\hline Facets at this vertex & $\sigma_{1}$ & $\sigma_{2}$ & $\sigma_{3}$ & $\sigma_{4}$ & $\sigma_{5}$ & $\sigma_{6}$ \\
\hline $12,23,34,45,56,16$ & + & + & + & + & + & + \\
\hline $15,16,56$ & + & + & + & + & + & - \\
\hline $45,46,56$ & + & + & + & + & - & + \\
\hline $34,35,45$ & + & + & + & - & + & + \\
\hline $23,24,34$ & + & + & - & + & + & + \\
\hline $12,13,23$ & + & - & + & + & + & + \\
\hline $12,16,26$ & - & + & + & + & + & \\
\hline $14,15,46,56$ & + & + & + & + & - & - \\
\hline $35,36,45,46$ & + & + & + & - & - & + \\
\hline $24,25,34,35$ & + & + & - & - & + & + \\
\hline $13,14,23,24$ & + & - & - & + & + & + \\
\hline $12,13,26,36$ & - & - & + & + & + & + \\
\hline $15,16,25,26$ & - & + & + & + & + & - \\
\hline $13,14,36,46$ & + & + & + & - & - & - \\
\hline $25,26,35,36$ & + & + & - & - & - & + \\
\hline $14,15,24,25$ & + & - & - & - & + & + \\
\hline $13,14,36,46$ & - & - & - & + & + & + \\
\hline $25,26,35,36$ & - & - & + & + & + & - \\
\hline $14,15,24,25$ & - & + & + & + & - & - \\
\hline $14,15,46,56$ & - & - & - & - & + & + \\
\hline $35,36,45,46$ & - & - & - & + & + & - \\
\hline $24,25,34,35$ & - & - & + & + & - & - \\
\hline $13,14,23,24$ & - & + & + & - & - & - \\
\hline $12,13,26,36$ & + & + & - & - & - & - \\
\hline $15,16,25,26$ & + & - & - & - & - & + \\
\hline $15,16,56$ & - & - & - & - & - & + \\
\hline $45,46,56$ & - & - & - & - & + & - \\
\hline $34,35,45$ & - & - & - & + & - & - \\
\hline $23,24,34$ & - & - & + & - & - & - \\
\hline $12,13,23$ & - & + & - & - & - & - \\
\hline $12,16,26$ & + & - & - & - & - & - \\
\hline $12,23,34,45,56,16$ & - & - & - & - & - & - \\
\hline
\end{tabular}

\section{OPTIMIZING THE TORQUE COMMANDS}

\section{Optimality Criterion}

Optimal use of the torque capability of a wheel configuration requires that equal demands be made on all wheels. In the case of wheels with identical capacities, this is accomplished by minimizing the maximum value, or $L_{\infty}$ norm, of the set of individual wheel torques which will produce the desired net torque. This minimax value $T_{\max }$, which must be determined, determines the torque envelope, as in the previous section, and the required torque commands; it clearly cannot exceed the wheel capability of any wheel. The generalization to wheel assemblies with varying capabilities is straightforward, but we will not consider this uncommon case. 


\section{Numerical Algorithm For Minimizing The $L_{\infty}$ Norm of a Torque Command}

Finding the $L_{\infty}$ norm requires identifying which facet of the wheel envelope contains a point along the desired torque axis. We can search for this facet by considering all pairs $i, j$ of wheels in turn until we find the correct facet. This procedure is quite general but is generally too slow for application onboard a spacecraft. The Swift spacecraft employs an algorithm tailored to the six-wheel configuration using the azimuth and elevation of the desired torque vector to determine the optimal facet via a rapidly executed series of inequalities. The following discussion applies to both strategies.

Define a scaled torque $n$-vector $\psi$ as

$$
\Psi \equiv T_{\max }^{-1} \mathbf{T}_{\text {wheels }},
$$

where $T_{\max }$ denotes the $L_{\infty}$ norm of the wheel torque vector, which is to be determined. It is clear from this scaling that the magnitude of the components of $\boldsymbol{\psi}$ is bounded by unity. Let the desired torque axis be represented by the unit vector $\hat{\mathbf{v}}$. Suppose, by previous knowledge or as a hypothesis, that the facet in the direction of $\hat{\mathbf{v}}$ has all wheels except those with indices $i$ and $j$ saturated. Then Eq. (6a) or (6b) immediately determines the magnitude and polarity of the other $n-2$ wheel torques. We tentatively assign a 1 or $a-1$ to each of these entries of $\psi$ according to Eq. (6a). If this is the correct assignment, there is some positive constant $\gamma$ such that

$$
\gamma \hat{\mathbf{v}}=T_{\max }^{-1} \mathbf{T}=T_{\max }^{-1} W \mathbf{T}_{\text {wheels }}=W \boldsymbol{\psi}=\sum_{k=1}^{n} \psi_{k} \hat{\mathbf{w}}_{k}
$$

which may be rearranged to

$$
\left[\begin{array}{lll}
-\hat{\mathbf{w}}_{i} & -\hat{\mathbf{w}}_{j} & \hat{\mathbf{v}}
\end{array}\right]\left[\begin{array}{c}
\psi_{i} \\
\psi_{j} \\
\gamma
\end{array}\right]=\sum_{k \neq i, j} \psi_{k} \hat{\mathbf{w}}_{k} \equiv \mathbf{z}
$$

If the matrix on the left side has full rank, the solution of this equation is

$$
\left[\begin{array}{c}
\psi_{i} \\
\psi_{j} \\
\gamma
\end{array}\right]=\frac{1}{\left(\hat{\mathbf{w}}_{i} \times \hat{\mathbf{w}}_{j}\right) \cdot \hat{\mathbf{v}}}\left[\begin{array}{c}
\left(\hat{\mathbf{w}}_{j} \times \hat{\mathbf{v}}\right) \cdot \mathbf{z} \\
\left(\hat{\mathbf{v}} \times \hat{\mathbf{w}}_{i}\right) \cdot \mathbf{z} \\
\left(\hat{\mathbf{w}}_{i} \times \hat{\mathbf{w}}_{j}\right) \cdot \mathbf{z}
\end{array}\right]
$$

A negative value of $\gamma$ means that the signs of Eq. (6b) should have been used rather than those of Eq. (6a), which is easily accomplished without repeating the computations by simply negating both $\gamma$ and $\psi$. The $L_{\infty}$ norm value $T_{\max }$ for a given desired torque is given by Eq. (10) as

$$
T_{\max }=T_{\text {desired }} / \gamma,
$$

and the individual wheel torques are given by Eq. (9). Clearly, the maximum torque that can be applied in the direction $\hat{\mathbf{v}}$ is $\gamma$ times the maximum torque per wheel.

If we had identified the appropriate facet for the desired torque direction a priori, as in the Swift onboard algorithm, the computation is complete. If we are searching over wheel pairs for the correct facet, it is possible either $\psi_{i}$ or $\psi_{j}$ has magnitude greater than unity or that the determinant $\left(\hat{\mathbf{w}}_{i} \times \hat{\mathbf{w}}_{j}\right) \cdot \hat{\mathbf{v}}$ is zero. This means that the facet corresponding to the pair $i, j$ is not the facet that determines the $L_{\infty}$ norm, so we must proceed to consider the next pair. We continue this search until we find a pair that gives an acceptable solution to Eq. (12).

A MATLAB algorithm implementing this procedure for determining $\psi$ is contained in the box below. This algorithm may be utilized in a loop to determine the wheel envelopes shown in Figures $1-4$ for configurations of 3, 4, 6, and 5 wheels. As illustrated by Figure 4, a single wheel failure in the six wheel case destroys the symmetry of the figure and significantly reduces the envelope. The maximum torque available in the direction of minimum capability drops by factor of 1.5 from the full six-wheel configuration. 


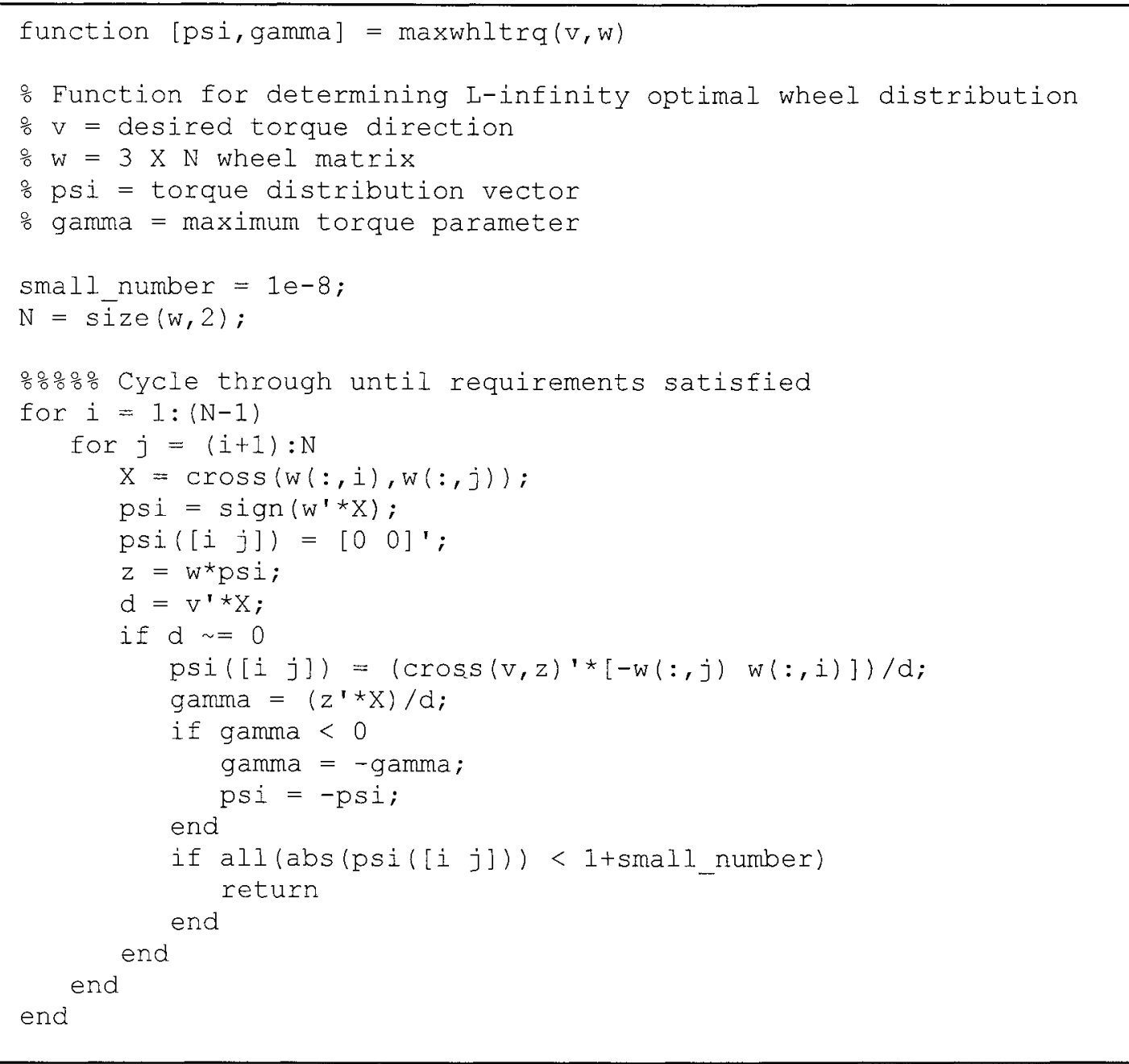

\section{CONCLUSIONS}

We have presented algorithms for determining the envelope of available torques and angular momenta in three dimensional space for general configurations of $n$ reaction wheels. These fill a polyhedron given by projection into three dimensional space of an $n$-dimensional hypercube in reaction wheel space. This polyhedron is the convex hull of the projection of the vertices of the hypercube, the points at which all the wheels are saturated in the positive or negative direction. We have also presented an algorithm for finding the optimal torque commands to produce any desired torque. The optimality criterion is that these commands minimize the maximum value, or $L_{\infty}$ norm, of the vector of individual wheel torques. The algorithm also determines the maximum torque available in any given direction. While the exact solution algorithm is not very efficient for onboard processing, it is applicable to arbitrary wheel configurations.

\section{REFERENCES}

1. http://swift.sonoma.edu/

2. Kawaguchi, J., Maeda, K., Matsuo, H., and Ninomiya, K., "Closed Loop Momentum Transfer Maneuvers Using Multiwheels," Journal of Guidance, Control, and Dynamics, Vol. 18, No. 4, July-Aug. 1995, pp. 867-874.

3. Steyn, W. H., "Near-Minimum-Time Eigenaxis Rotation Maneuvers Using Reaction Wheels," Journal of Guidance, Control, and Dynamics, Vol. 18, No. 5, Sept-Oct. 1995, pp. 1184-1189.

4. Alonso, Roberto, and Crassidis, John, "Reaction Wheel Configuration Analysis," submitted to the Journal of Guidance, Control, and Dynamics 


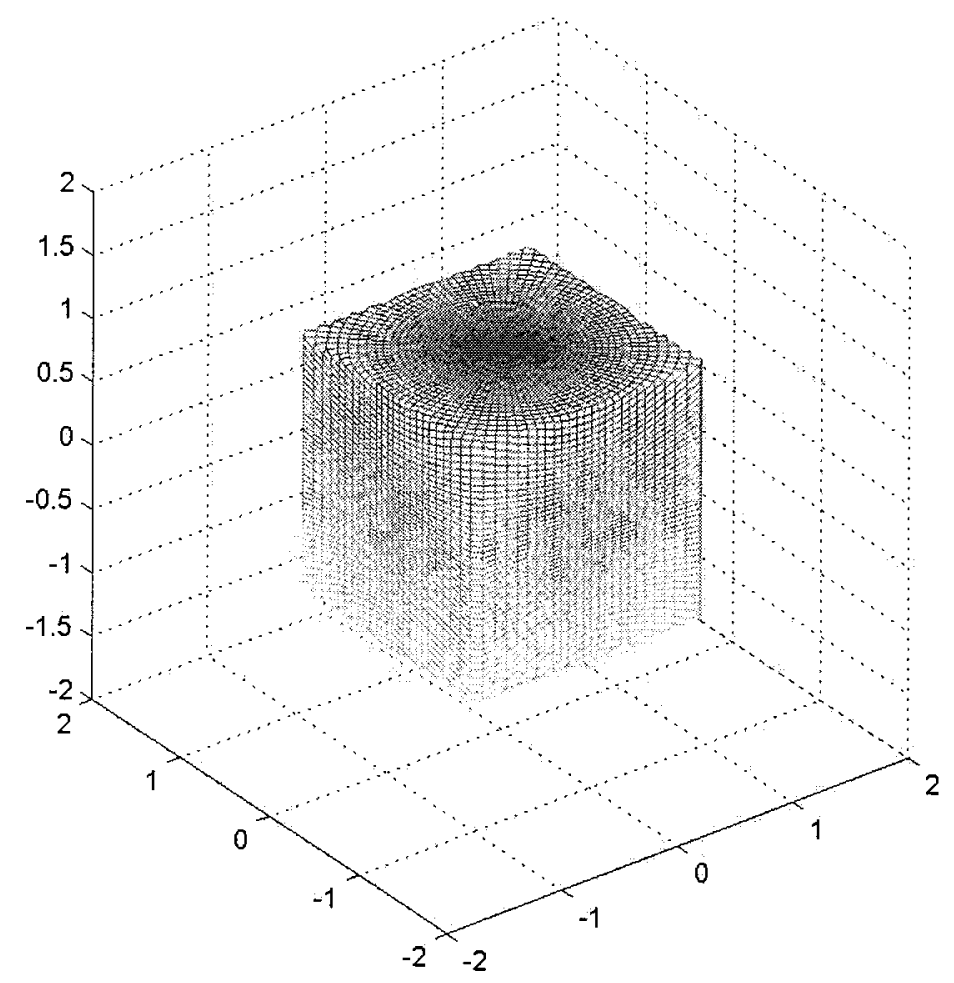

Figure 1. Three Orthogonal Wheel Configuration Envelope

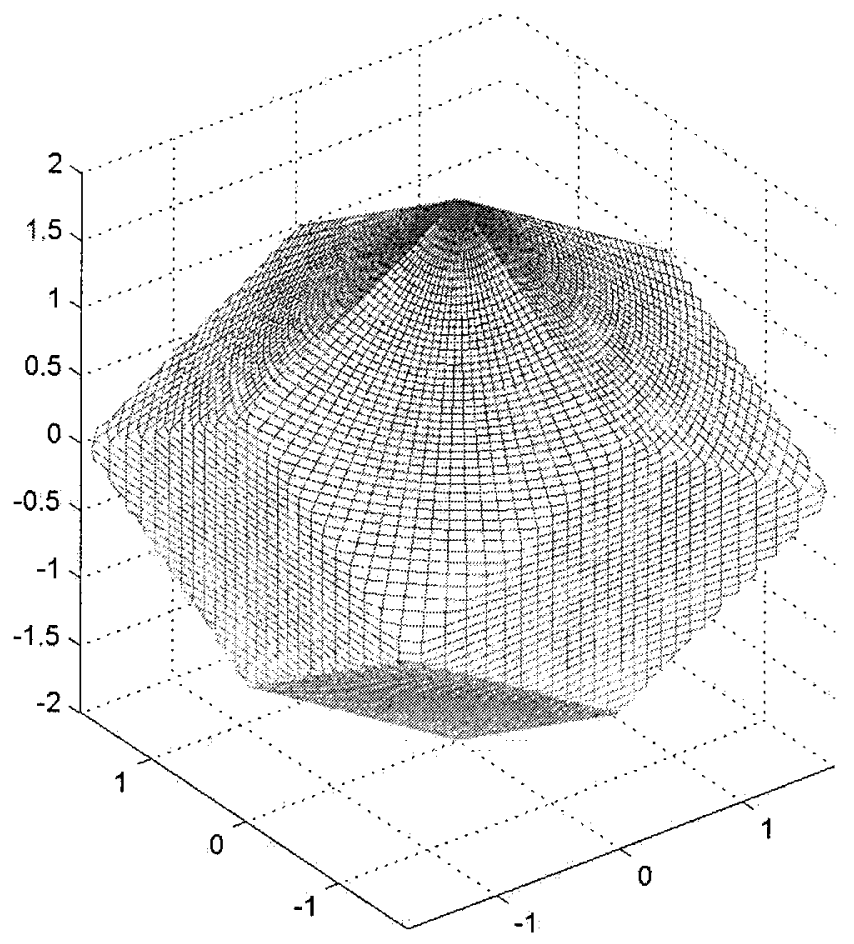

Figure 2. Four Symmetric Wheel Configuration Envelope 


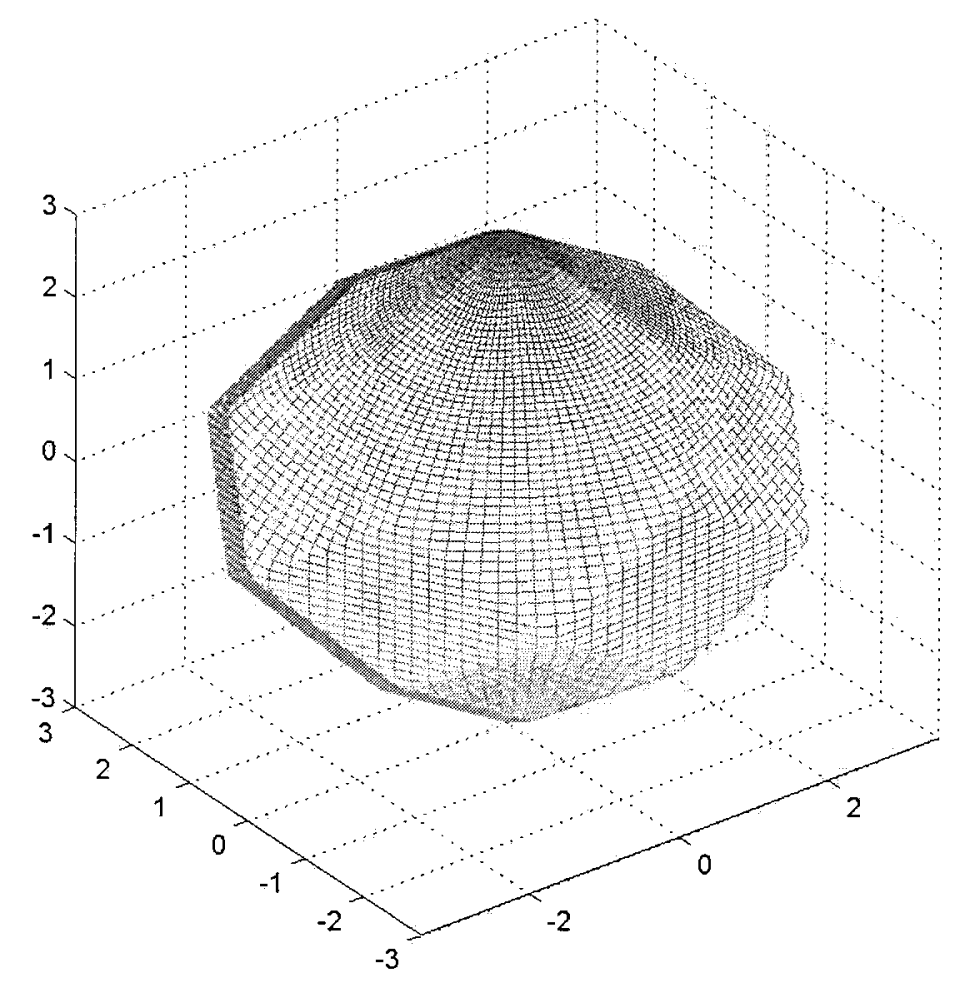

Figure 3. Six Symmetric Wheel Configuration Envelope

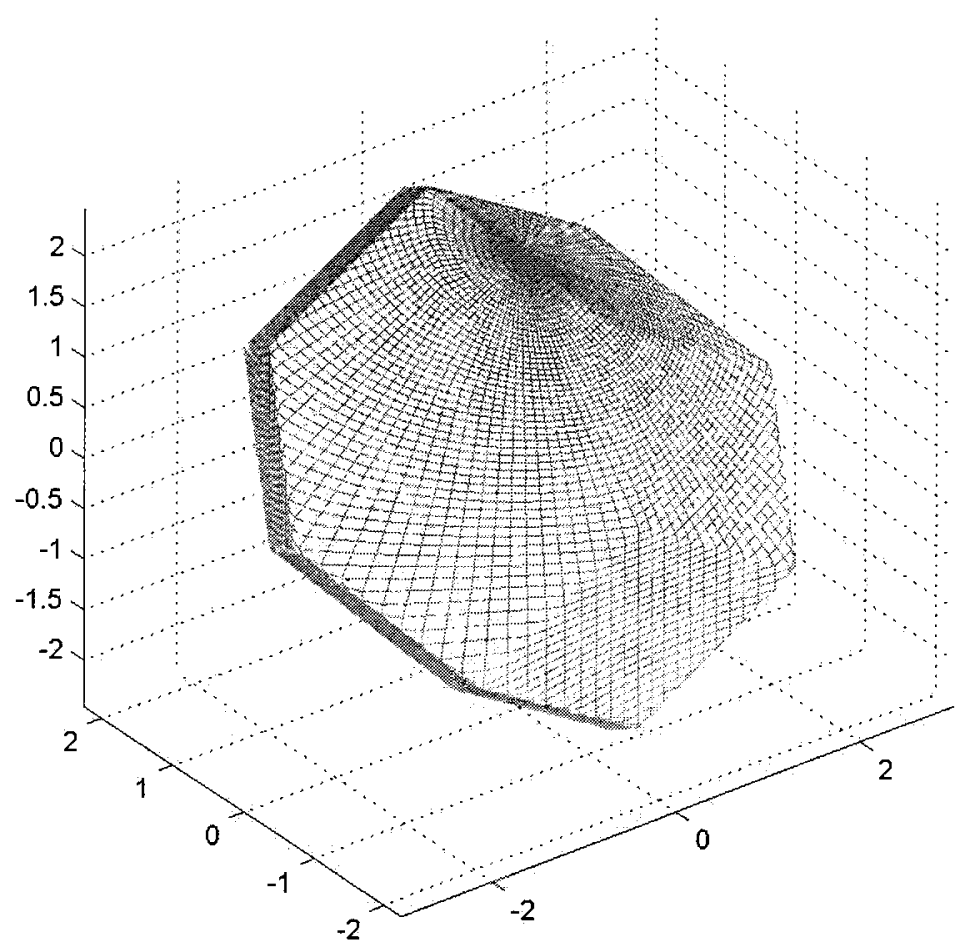

Figure 4. Envelope of Six Wheel Configuration with One Wheel Failed 